\title{
Echecs de marchés et pauvreté : l'exemple de la filière cacao en Côte d'Ivoire
}

Oléagineux, Corps Gras, Lipides. Volume 8, Numéro 6, 577-83, Novembre - Décembre 2001, Dossier : L'avenir des cultures pérennes

Auteur(s) : Catherine ARAUJO BONJEAN, Gérard CHAMBAS, Jean-Louis COMBES, CERDI - Université d'Auvergne, 65, boulevard François-Mitterrand, 63000 Clermont-Ferrand, France.

Résumé : La question posée ici est donc de savoir si, dans une économie où les défaillances de marché sont multiples, le désengagement de l'État a conduit à une amélioration du bien-être des planteurs, plus particulièrement des pauvres, ou non. Nous montrons, dans un premier point, que la libéralisation a entraîné une rupture dans le mode de coordination des agents au sein de la filière, rupture dont les conséquences sur la pauvreté sont ensuite examinées. Quatre critères de pauvreté sont considérés [2]: le niveau de revenu, la vulnérabilité face au risque de revenu, l'accès aux biens et services publics et la participation des paysans aux décisions au sein de la filière.

Summary: Given the importance of market failures in Côte d'Ivoire, the question arises to know whether the liberalisation process in the cocoa sector led to an increase in producers' welfare, and especially in the poorest. We show that a major consequence of the liberalisation was a sharp decline in forward transactions on the international market and at collect level. This phenomenon led to an increase in producers' vulnerability, as their exposure to income risk rose dramatically and the range of risk-coping instruments was narrowed. Because of their low capacity to save, the absence of any insurance mechanism and an underdeveloped credit market, households were not able to smooth their consumption and had to cut down some expenditure. Scholarship expenses, which occur before the beginning of the cocoa season, were especially affected. Besides, the liberalisation was followed by a significant decrease in the role played by the co-operatives.

Keywords: cocoa, Côte d'Ivoire, poverty, contract, market failures.

\section{ARTICLE}

\section{Libéralisation et mode de coordination des agents : la suspension des transactions en différé}

Avant 1999, l'intervention de l'État permettait aux opérateurs situés à des stades différents de la filière (paysans et sociétés de commerce d'une part, exportateurs et importateurs d'autre part) de s'engager dans des accords de coopération durables, palliant ainsi certaines inefficacités du marché. La libéralisation a entraîné un changement radical du mode de coordination des agents qui sont maintenant contraints d'effectuer l'ensemble de leurs transactions au comptant.

Évolution du mode d'organisation de la filière

La production de cacao en Côte d'Ivoire s'est développée dans le cadre d'une filière administrée où la Caisse de soutien et de stabilisation des prix des produits agricoles (CSSPPA) jouait un rôle central. Elle fixait en début de campagne un prix garanti au producteur, identique sur l'ensemble du territoire, et contrôlait la commercialisation extérieure des produits à travers la distribution de quotas d'exportation aux exportateurs agréés. La commercialisation intérieure était assurée par le secteur privé mais l'ensemble des coûts et des marges de commercialisation, depuis le prix d'achat bord 
champ au producteur jusqu'au prix garanti à l'exportateur, était fixé dans un barème. La Caisse percevait un prélèvement variable, égal à la différence entre le prix de réalisation à l'exportation et le coût de revient CAF (coût, assurance et frêt) fixé dans le barème.

Ce système de stabilisation était à l'origine de nombreuses inefficacités. D'une part, le mécanisme de stabilisation, détourné de ses objectifs initiaux, a été utilisé comme un instrument fiscal et la prédominance de l'objectif de recette a conduit à maintenir le prix payé au producteur à un niveau en moyenne très inférieur au prix international. De plus, en dérogeant aux procédures budgétaires habituelles, le système d'affectation des ressources de la Caisse de stabilisation a introduit des facteurs supplémentaires de déséquilibre et d'inefficacité dans l'utilisation des ressources publiques $[3,4]$. D'autre part, le mécanisme du barème, dont certains postes étaient largement surestimés, et le système de gestion des quotas d'exportation ont contribué au développement de sociétés de commerce bénéficiant de situation de rente.

Les premières réformes sont intervenues au début des années 90 après la " guerre du cacao ${ }^{1}$ ". Ainsi, en 1990, les pouvoirs publics abandonnent l'objectif de stabilisation pluriannuelle des prix mais la Caisse continue à gérer un mécanisme de régulation des ventes à l'exportation qui lui permet encore de fixer par avance, mais pour la durée de la campagne seulement, l'ensemble des prix en amont de la filière. Ce mécanisme de stabilisation intra-annuelle repose sur un programme de ventes à l'exportation, le PVAM (Programme de ventes anticipées à la moyenne), qui doit permettre de vendre au prix moyen du marché, sans chercher à réaliser un prix supérieur à la moyenne. Les droits d'exportation (déblocages) sont, dans une première période, attribués par la Caisse puis, à partir de mai 1996, mis aux enchères auprès des exportateurs agréés. L'achat d'un déblocage garantit un prix FOB (Free on Board) pour l'exportateur. Si le prix de vente (prix d'exécution du contrat) excède le prix FOB garanti, l'exportateur reverse la différence à la Caisse ; dans le cas inverse, il bénéficie d'un soutien $[5,6]$.

\section{Libéralisation et arrêt des transactions différées}

La libéralisation instaure un nouveau mode d'organisation de la filière caractérisé, notamment, par l'apparition d'acteurs privés d'envergure mondiale (ADM, Cargill). Ces groupes cherchent à assurer leurs approvisionnements en s'implantant directement dans les pays producteurs. Pour conquérir des parts de marché, ils se livrent une forte concurrence à l'achat du produit, rendant impossible, en l'absence d'un cadre juridique bien défini, le respect des engagements à terme au stade de l'exportation et de la collecte.

\section{* Transactions au stade de l'exportation}

Avant 1999, l'État ivoirien, à travers la caisse de stabilisation, jouait un rôle crucial en imposant une entente entre les exportateurs privés pour la mise en marché du produit. Le mécanisme de stabilisation (PVAM) permettait à l'exportateur, à travers l'achat de droits d'exportation, de couvrir ses ventes et de s'engager à terme vis-à-vis des acheteurs internationaux. Dans ce système de vente à l'exportation, obligatoire pour tous les exportateurs, la Caisse de stabilisation était garante du respect des engagements pris par les exportateurs privés, de sorte que le risque de contrepartie était considérablement réduit. De fait, la Caisse était considérée sur le marché international comme un intervenant fiable et pouvait vendre longtemps à l'avance, parfois jusqu'à 18 mois avant la récolte [5, $6]$. 
Le contrôle des ventes prenait la forme d'une autorisation préalable d'exportation avec vérification de la conformité du prix obtenu au prix mondial et octroi d'un quota d'exportation. Le contrôle du prix permettait de lutter contre la sous-facturation et le système de gestion des quotas équivalait à un mécanisme de caution solidaire, dans la mesure où il permettait de faire face à la défaillance d'un exportateur. Cette intervention débouchait sur la publication d'un prix indicatif au producteur annoncé en début de campagne, dont la crédibilité était assurée puisqu'il découlait du prix effectif de réalisation à l'exportation.

Avec la libéralisation, les ventes anticipées se sont effondrées en raison de l'importance du risque de non-livraison. En effet, dans un système libéralisé, les exportateurs peuvent être dans l'impossibilité de respecter leurs engagements s'ils n'ont pas le produit en magasin au moment voulu ou en l'absence de contrat à terme avec les producteurs. Or, dans un environnement caractérisé par une forte concurrence à l'achat et par la défaillance du système juridique, les exportateurs sont dans l'impossibilité de sécuriser leurs approvisionnements à travers des accords commerciaux avec les paysans. De fait, le respect des engagements entre un exportateur et une multitude de petits producteurs, ou de coopératives, tentés de vendre au plus offrant, est aujourd'hui impossible à garantir.

\section{* Transactions au stade de la collecte}

La régulation des exportations par la Caisse favorisait aussi l'émergence de relations de coopération, fondées sur la confiance, entre paysans et sociétés de commerce. Au stade de la collecte, la concurrence par les prix était fortement réduite de même que les comportements opportunistes des paysans et des sociétés de commerce, en l'absence d'un véritable État de droit. Les paysans pouvaient alors, par un engagement contractuel le plus souvent implicite avec une société de commerce, accéder à certains biens pour lesquels le recours au marché engendre des coûts de transaction prohibitifs, la transaction étant dénouée à la récolte.

Le principal intérêt des producteurs pauvres à s'engager dans une relation contractuelle avec une société industrielle ou commerciale était d'avoir un accès plus facile à un crédit de court terme. Le contrat fixait alors les conditions d'accès et de remboursement du crédit fourni par la société de commerce à travers le mécanisme des " avances en brousse ». Le contrat entre paysans et sociétés de commerce comporte des éléments proches du contrat commercial à terme. Ce contrat, établi avant la récolte, fixe les conditions de remboursement des emprunts et de vente du produit (prix, qualité, quantité) et, éventuellement, les délais de livraison. D'une façon générale, la société se réserve le droit de refuser les produits de mauvaise qualité ou de les déclasser. Cependant, ce type de contrat va audelà du simple contrat commercial dans la mesure où la répétition des transactions et l'apport de crédit introduisent un élément de coopération durable que l'on retrouve dans des filières quasi intégrées [7].

En favorisant une vive concurrence entre les exportateurs, la libéralisation a entrainé une augmentation importante du risque de défaut des partenaires et l'arrêt des transactions différées au stade de la collecte, en particulier du mécanisme de préfinancement des achats de cacao. En raison du délai entre le moment où le paysan s'engage vis-à-vis d'une société et la récolte, la transaction est soumise à deux catégories d'incertitude : une incertitude externe, liée aux variations exogènes de l'offre (aléas climatiques) et des prix mondiaux, et une incertitude interne, liée à l'effort inobservable 
des paysans qui peuvent ainsi justifier leur absence de livraison. Ces deux sources d'incertitude sont à l'origine d'asymétries informationnelles et de comportements opportunistes de la part des paysans et des sociétés de commerce. Ainsi, par exemple, en cas de chute des cours mondiaux, la société de commerce, qui s'est engagée à acheter à prix fixe la récolte des producteurs, sera tentée de ne pas respecter ses engagements. Inversement, en cas de hausse des prix mondiaux, les paysans sont incités à tricher en recherchant le meilleur offreur.

D'une façon générale, au stade de la collecte comme au niveau de l'exportation, la libéralisation pose le problème essentiel du respect des engagements lorsque les institutions juridiques sont défaillantes. On considère généralement que le respect des engagements est assuré par les effets de réputation. Or, le caractère répété des engagements n'assure pas nécessairement l'émergence d'une solution coopérative entre les agents car la menace de non-reconduction de l'engagement n'est généralement pas crédible. De plus, dans les pays en développement, les agents, notamment les pauvres, ont une préférence élevée pour le présent, qui les pousse à ne pas respecter leurs engagements.

\section{Libéralisation et revenu des planteurs}

Alors que les producteurs attendaient, pour la première campagne libéralisée, une augmentation de leur pouvoir d'achat, leur revenu a chuté de 50 \% par rapport à la campagne précédente, en raison principalement de la chute des cours mondiaux mais aussi de la faible productivité des exploitations.

\section{Chute des prix à la production}

Les prix au producteur ont été divisés par deux entre 1998/1999 et 1999/2000, passant d'une moyenne de $547 \mathrm{FCFA} / \mathrm{kg}$ à $275 \mathrm{FCFA} / \mathrm{kg}$; en valeur réelle, ces prix sont à leur plus bas niveau depuis 1960. La chute des prix au producteur est la conséquence de la baisse des cours mondiaux (figure) que ne pouvait compenser la réduction des coûts et des marges de commercialisation.

\section{* Chute des cours mondiaux du cacao}

La libéralisation de la filière ivoirienne, anticipée dès le début de la campagne 1998/1999, a vraisemblablement contribué de façon transitoire à la baisse des prix mondiaux amorcée dès juin 1998. En novembre 1998, les premières défaillances de certains exportateurs conduisent en effet le marché à anticiper des disponibilités importantes. Ces anticipations sont renforcées par les perspectives de libéralisation et jouent dans le sens d'une baisse des cours. Fin 1999, les prévisions de récolte ivoirienne révèlent un niveau de production record tandis que la consommation mondiale régresse. Cette détérioration des fondamentaux du marché accentue alors la chute des cours.

Par ailleurs, du fait de l'abandon du mécanisme de ventes anticipées, les exportateurs ne peuvent bénéficier, en situation normale, des reports de marché, ce qui a pu contribuer à la faiblesse des prix de vente à l'exportation ${ }^{2}$. Enfin, on peut attendre de l'arrêt des ventes à terme une augmentation de la variabilité intra-annuelle des cours liée aux fluctuations saisonnières de la production ivoirienne, ce que semble montrer la remontée des cours au premier semestre 2001.

\section{* Efficacité accrue des circuits commerciaux}

Le système d'incitation, qui résultait de l'administration des coûts et marges de commercialisation intérieurs à travers un barème, était considéré comme inefficace et défavorable aux planteurs. En effet, l'État ne dispose généralement pas des informations sur les opérateurs privés qui lui permettraient d'établir ces prix à leur niveau de concurrence. De plus, l'État peut, à travers la fixation 
des coûts et marges de commercialisation, chercher à favoriser les industriels et exportateurs au détriment des paysans. Aujourd'hui encore, il semblerait que la tarification d'un certain nombre de services imposés aux exportateurs (contrôle de qualité à l'embarquement, certification des exportations, etc.) soit surévaluée du fait de la situation de monopole de certains prestataires de services [7].

Néanmoins, les paysans ont bénéficié, dès la première campagne libéralisée, de la concurrence introduite par de nouveaux opérateurs qui se sont engagés dans une course au produit en pratiquant une surenchère sur les prix d'achat des fèves. Les coûts de commercialisation intérieure ont baissé de $35 \%$ en valeur courante et en valeur réelle sur la première campagne libéralisée ${ }^{3}$. Ils restent toutefois, en valeur réelle, à un niveau relativement élevé. Ainsi, si l'on compare le niveau des coûts de commercialisation en 1982 et 1999, années où le prix mondial est au même niveau (520 FCFA/kg), on constate une forte progression des coûts et marges des intermédiaires de la filière $(+71 \%)$ au détriment des producteurs (- $8 \%$ ) et de l'État (- $11 \%)$.

\section{* Élimination des opérateurs nationaux moins efficaces}

La diminution des marges commerciales a entraîné l'éviction des opérateurs nationaux les moins compétitifs. La capacité des grands groupes à surenchérir s'explique à la fois par des coûts de fonctionnement moins élevés et par l'acceptation de marges plus faibles dans une stratégie de conquête de marché.

Par rapport aux exportateurs traditionnels, les grands groupes bénéficient, du fait de leur appartenance à des multinationales, d'économies d'envergure notamment au niveau du fret. Ainsi, les sociétés qui peuvent affréter leur propre cargo et transporter le cacao en vrac réalisent des économies substantielles sur le fret. Surtout, les frais financiers de ces nouveaux opérateurs sont considérablement réduits par rapport à ceux des anciennes sociétés de la place. D'une part, ils n'assurent pas de préfinancement de leurs achats et paient le produit au comptant. D'autre part, les filiales de multinationales ont la possibilité de mobiliser des financements internes au groupe ou de se financer sur les marchés internationaux de capitaux, ce qui réduit leurs frais financiers par rapport aux opérateurs contraints de se financer auprès des banques locales ${ }^{4}$. Enfin, la transformation locale du cacao leur permet de bénéficier d'avantages fiscaux et d'un droit de sortie à taux réduit sur les produits semi-transformés. Ces avantages ainsi que la nécessité d'approvisionner les usines incitent les industriels à pratiquer des prix d'achat plus élevés.

\section{Faible productivité des exploitations}

La productivité des exploitations dépend étroitement des soins apportés aux plantations et notamment des traitements phytosanitaires effectués. Or, au début de la campagne 2000/2001, avant les troubles socio-politiques qui ont marqué le pays, les planteurs avaient employé moins de maind'œuvre saisonnière et réduit les traitements phytosanitaires, ce qui a contribué à la baisse des rendements [8]. Le premier traitement des cacaoyers, déterminant pour le rendement futur et qui a lieu normalement en juillet-août, a été particulièrement affecté. Ce phénomène peut être mis en relation avec la chute des revenus sur deux campagnes consécutives et avec les difficultés d'accès au crédit des planteurs.

Les planteurs, notamment les pauvres, sont soumis à une forte contrainte de liquidités en raison de leur faible niveau de revenu et d'un accès aujourd'hui plus limité aux circuits de crédit faute de collatéraux et d'une offre de crédit adéquate : la Banque nationale de développement agricole a été 
liquidée, l'activité du Crédit mutuel reste limitée et le crédit de campagne s'est effondré.

La productivité des plantations et la qualité des fèves dépendent aussi de la diffusion des nouvelles technologies et du conseil agricole. Or, la dégradation des services publics d'encadrement liée aux difficultés financières de l'État ainsi que l'impuissance des pouvoirs publics à garantir le respect de normes de qualité pour les intrants agricoles contribuent à la mauvaise qualité des intrants disponibles et à la faiblesse des rendements ${ }^{5}$. Face à ces défaillances de l'État, les sociétés privées de commercialisation ne peuvent prendre le relais que si elles sont assurées de bénéficier des retombées positives de leur investissement dans le conseil agricole ou la fourniture d'intrants. Cela supposerait qu'elles soient en mesure de contrer les comportements opportunistes des paysans en disposant d'une situation de monopole sur leur zone de collecte ${ }^{6}$ ou en intégrant l'activité de production.

\section{Vulnérabilité des paysans face au risque de revenu}

Les planteurs, qui n'avaient jamais connu d'autre situation que celle d'un prix unique et stabilisé, se trouvent aujourd'hui confrontés à un phénomène nouveau : l'instabilité intra-annuelle des prix du cacao. En l'absence de tout système d'assurance, les paysans et notamment les pauvres n'ont que très peu de moyens de se protéger des conséquences néfastes du risque engendré par l'instabilité des prix. Ils sont dès lors incités à diversifier leurs productions et contraints de réduire leurs dépenses de consommation courante en période de soudure.

\section{Exposition accrue au risque de revenu}

Malgré la mise en place d'un système d'information sur les prix assuré par les médias, le PRIMAC (Programme d'information sur les marchés du café-cacao), les paysans sont peu ou mal informés de l'évolution des marchés locaux et internationaux ${ }^{7}$. De plus, le cacao ne pouvant être conservé sur l'exploitation, les planteurs ne peuvent jouer sur les stocks pour réguler leur offre en fonction de leurs anticipations de prix. Ils ne sont donc pas en mesure de tirer profit de hausses futures des prix et sont contraints de vendre dès la récolte.

Surtout, les producteurs de cacao ont été confrontés à la gestion des risques de prix et de revenu dans un contexte particulièrement défavorable, caractérisé par la succession de deux années de très bas prix, qui a conduit à un tarissement de leur épargne. Face à cet épuisement de leurs réserves, les ménages n'ont pu se tourner vers les circuits formels de crédit qui restent peu actifs en zone rurale, voire inexistants dans certaines régions. Mais la libéralisation a également eu des conséquences directes sur l'accès des ménages aux circuits informels du crédit.

D'une part, la libéralisation a entraîné l'arrêt du système de préfinancement des achats de cacao par les exportateurs. Les paysans qui pouvaient ainsi bénéficier d'un crédit de quelques semaines, pour financer certaines de leurs dépenses courantes avant le début de la campagne, ont été pénalisés par la suspension de cette forme de crédit ${ }^{8}$. Dans la structure actuelle du marché, ils ne peuvent pas non plus espérer, à travers un engagement contractuel avec une société de commercialisation, transférer le risque de prix à l'opérateur commercial. Le risque de défaut du paysan est actuellement trop grand pour qu'une société s'engage, avant la récolte, à acheter le produit à un prix fixé à l'avance.

D'autre part, le risque de prix a entraîné une raréfaction des sources de crédit traditionnelles en milieu rural : famille, amis, commerçant local, etc. En effet, dans le système de prix stabilisés, le revenu futur de l'emprunteur pouvait être assez facilement évalué, et sa variabilité, essentiellement due aux aléas climatiques, était suffisamment faible pour permettre de sécuriser le prêt. En entraînant une 
dévalorisation du collatéral que représente la récolte future de cacao, l'instabilité intra-annuelle des prix a eu pour conséquence une réduction de l'offre de crédit ${ }^{9}$.

\section{Risque de consommation et diversification de la production}

Ainsi, en l'absence d'épargne, de mécanisme d'assurance et d'un système de crédit développé, le risque de revenu s'est répercuté sur la consommation des ménages, engendrant une contraction de la dépense, notamment des dépenses d'éducation, en période de soudure. Bien évidemment, les producteurs les plus pauvres sont plus particulièrement affectés. De fait, une réduction sensible de certaines dépenses courantes de consommation a pu être constatée au troisième trimestre 2000. Les dépenses de scolarité, qui interviennent normalement en septembre, avant le début de la campagne cacaoyère, sont particulièrement touchées et les retards de scolarisation ont été nombreux lors de la dernière rentrée.

Il en résulte aussi un changement sensible du comportement des producteurs qui investissent moins dans leurs plantations et cherchent à diversifier leurs sources de revenu. Les producteurs de cacao se sont engagés dans des processus de diversification de leurs sources de revenu ou ont renforcé des stratégies déjà amorcées dans les années 90 . Ils investissent notamment dans l'hévéa qui présente l'avantage de fournir des revenus certes plus faibles que ceux du cacao mais échelonnés tout au long de l'année. Dans les zones favorables, ils investissent également dans le palmier à huile. Malgré un coût d'investissement et un délai de production importants pour ces cultures pérennes, les producteurs considèrent que leurs revenus sont plus sûrs ${ }^{10}$.

Au total, plusieurs facteurs peuvent expliquer la chute de la production de cacao en 2000/2001, chute estimée à environ 300000 tonnes, parmi lesquels le faible niveau des prix et le risque de revenu jouent un rôle important. Outre une mauvaise pluviométrie et un contexte socio-politique hostile aux exploitants immigrés, les paysans à court d'épargne et n'ayant pas accès au crédit ont moins investi dans leurs plantations.

\section{Libéralisation et redistribution publique}

Compte tenu de l'importance de la contribution de la filière cacao au budget de l'État ivoirien et du lien étroit, en longue période, entre le niveau des ressources publiques et l'offre de biens publics, le prélèvement sur la filière cacao est un élément important du financement des biens publics. La structure et le niveau du prélèvement sur la filière cacao sont eux-mêmes étroitement liés à son mode d'organisation.

\section{Fiscalité sur les exportations}

Les taxes sur les produits exportés demeurent une source essentielle de recettes publiques, tandis que la fiscalité sur les bénéfices des sociétés de commerce et de transformation reste relativement faible.

Jusqu'en 1985, le droit unique de sortie (DUS) représentait une faible part du prélèvement total opéré sur la filière, qui consistait principalement en des prélèvements de la Caisse de stabilisation. Ces derniers sont devenus négligeables à partir de 1990, du fait de l'abandon de l'objectif de stabilisation inter-annuelle des prix. Le DUS, supprimé entre 1989 et 1993 à la suite de la baisse des cours mondiaux, a été réintroduit en 1994 au moment de la dévaluation du franc CFA et fixé à un niveau élevé (200 FCFA $/ \mathrm{kg})^{11}$. Il est, depuis, fortement corrélé avec la valeur du prix mondial exprimé en 
FCFA $^{12}$, de sorte que le montant du prélèvement total sur les exportations de cacao, pour un même prix international, n'a pas sensiblement changé depuis l'abandon de la stabilisation des prix.

Cette stratégie de maintien d'un niveau élevé de prélèvement à l'exportation se justifie par la place dominante de la Côte d'Ivoire sur le marché mondial. De ce fait, l'incidence de la taxe à l'exportation est en grande partie supportée par les consommateurs étrangers. Ainsi, comme cela a pu être observé en $1999^{13}$, une baisse du DUS profiterait vraisemblablement davantage aux consommateurs étrangers, sous forme d'une baisse du prix international, qu'aux producteurs ivoiriens.

\section{Redistribution et offre de biens publics}

Certaines formes d'intervention sur les prix peuvent être souhaitables du point de vue de l'équité si elles bénéficient aux pauvres. C'est le cas, en principe, des politiques de stabilisation des prix qui doivent permettre d'accroître le bien-être des plus pauvres dont l'aversion au risque est plus grande et les moyens de protection contre le risque particulièrement limités. C'est aussi le cas de la péréquation spatiale des prix dont on attend un effet positif sur le revenu des paysans pauvres. En allant à l'encontre d'une spécialisation régionale, la péréquation des prix est inefficace mais la fixation d'un prix unique sur l'ensemble du territoire constitue un instrument de redistribution au profit des paysans les plus éloignés des marchés qui sont généralement ceux des régions les plus pauvres.

Ces deux formes d'intervention, péréquations spatiale et temporelle des prix, peuvent donc être considérées comme des instruments de redistribution à travers le système des prix, au bénéfice des plus pauvres. Bien évidemment, un système de subventions directes au profit des pauvres et aussi forfaitaires que possible serait préférable du point de vue de l'efficacité, car n'interférant pas avec les mécanismes de marché. Cependant, compte tenu de la situation des finances publiques de la Côte d'Ivoire, de la capacité administrative et des coûts d'administration, un tel système n'est pas envisageable actuellement.

Par ailleurs, la politique de prix garantis et stabilisés telle qu'elle a été pratiquée en Côte d'Ivoire jusqu'à la fin des années 80 peut être considérée comme un système de redistribution des producteurs de cultures de rente (principalement café, cacao et coton) au profit des classes urbaines (fonctionnaires, employés des sociétés publiques, etc.). Cependant, malgré l'importance des prélèvements publics sur les cultures de rente, la politique de prix a certainement constitué un facteur de cohésion sociale entre groupes sociaux et régions différentes. Ainsi, les prélèvements sur l'agriculture d'exportation ont servi à financer une politique de rééquilibrage en faveur du Nord, d'importantes infrastructures publiques, aussi bien en zone urbaine que rurale, ainsi qu'un système éducatif développé.

\section{Libéralisation et participation des planteurs pauvres}

Les mesures d'accompagnement de la libéralisation prévoyaient la restructuration et le renforcement du mouvement coopératif ainsi que la participation des paysans au processus de décision dans la filière cacao. Ces mesures devaient notamment permettre aux paysans de faire prévaloir leurs intérêts dans la définition de la politique cacaoyère en renforçant leur pouvoir de négociation vis-à-vis des sociétés de commerce et de l'administration. 
La libéralisation devait s'accompagner du renforcement du rôle des organisations de producteurs pour leur permettre d'intervenir jusqu'à l'exportation du produit. La transformation des GVC (Groupement villageois à vocation coopérative) en coopératives était une première étape de ce processus qui devait permettre d'assainir les groupements existants en leur imposant de nouveaux modes d'organisation et de gestion ${ }^{14}$. II s'avère en fait que la libéralisation a entraîné une réduction sensible du rôle des nouvelles coopératives : la part des produits collectés par les GVC et coopératives est estimée à $25 \%$ sur la campagne 1999/2000 contre $32 \%$ en 1998/1999 [9].

Cette réduction de l'activité des coopératives est en grande partie imputable à leurs difficultés de financement qui les ont conduit à développer leurs achats à crédit auprès des paysans (ils passent de $15 \%$ en 1998/1999 à $32 \%$ en 1999/2000) alors que, dans le même temps, les achats à crédit effectués par le secteur privé baissaient de 6 à $2 \%$. Le délai de paiement des producteurs par la coopérative est en général de plusieurs jours, le temps que celle-ci livre son produit au magasin de l'exportateur ou à l'usine. L'exportateur, lui, paie comptant à la livraison, à la coopérative ou au traitant.

Le mouvement coopératif reste donc largement minoritaire parmi les producteurs de cacao ${ }^{15}$ et a été plutôt affaibli par la libéralisation. En effet, en l'absence de fonds propres et d'accès au crédit bancaire, les coopératives, contraintes d'acheter à crédit dans un contexte où toutes les transactions s'effectuent au comptant et où les paysans ont un besoin pressant de liquidités, voient leurs parts de marché diminuer. De plus, les nouveaux opérateurs commerciaux tendent à pratiquer une politique de prix discriminante vis-à-vis des groupements de producteurs. Par le passé, l'exportateur accordait une prime à la coopérative en rémunération des services de collecte, ce qui ne semble plus être le cas aujourd'hui ; bien souvent, le prix payé à la coopérative au stade entrée usine n'est pas différent du prix payé au paysan.

\section{Risque d'exclusion des pauvres}

En traitant avec des groupements de producteurs, les firmes devraient pouvoir réduire significativement leurs coûts de fonctionnement (coûts de négociation d'un nombre plus réduit de contrats, de surveillance, de collecte des produits et de fourniture de biens et services annexes) et devraient donc être incitées à encourager leur développement. Cependant, les organisations paysannes peuvent aussi être perçues comme une menace par les sociétés de commerce, voire par l'État lui-même, dès lors qu'elles sont susceptibles de négocier collectivement, voire d'évoluer vers un mouvement syndical et de constituer une force organisée.

Le risque est aussi que les paysans pauvres soient marginalisés dans les relations sociétés de commerce/planteurs. L'expérience montre en effet que les sociétés de commerce préfèrent contracter avec un nombre réduit de grands cultivateurs plutôt qu'avec un grand nombre de petits paysans [10].

D'une façon générale, une société privée de commerce ou de transformation à la recherche du profit maximum préférera traiter avec les paysans les plus aisés, qui sont généralement les plus proches des axes de communication, disposant de grandes exploitations et d'un bon niveau d'éducation. L'intérêt de la firme est en effet de traiter avec un nombre limité de grands producteurs afin de réduire les coûts de négociation des contrats mais aussi les coûts fixes associés à la fourniture de certains produits et services : crédit, intrants, conseil technique, encadrement, etc. Les grandes exploitations ont pour autre avantage d'offrir une production plus importante et de qualité homogène. 
Par ailleurs, le risque de défaut d'un paysan (non-remboursement du crédit ou non-livraison des produits) est généralement plus faible pour un paysan disposant d'un capital d'exploitation important. Compte tenu de la difficulté pour une société, et notamment une société étrangère, de sanctionner les paysans qui ne respectent pas leurs engagements, elle préférera donc traiter avec ceux qui présentent davantage de garanties. De même, on s'attend à ce que les paysans les plus riches soient aussi ceux dont le niveau d'éducation est le plus élevé. Leur plus grande capacité à adopter de nouvelles technologies permettra alors à la firme de réduire ses coûts de vulgarisation, d'encadrement et de contrôle.

Enfin, les sociétés sont souvent réticentes à s'engager dans des activités autres que la commercialisation et la transformation des produits. Or les petits producteurs sont ceux qui ont le plus de difficultés à accéder aux marchés et, par conséquent, ce sont ceux qui demandent à la firme le plus d'intrants, de capital et de services d'assistance par unité de produit [11]. Les coûts associés aux activités de crédit, de distribution d'intrants et d'assurance sont généralement élevés et les firmes peuvent avoir des difficultés à les répercuter en intégralité sur les paysans. Cela impliquerait en effet que la firme puisse offrir un contrat différent, et donc des prix différents, à chaque cultivateur en fonction de ses besoins spécifiques en intrants et en services annexes. II en résulterait alors une augmentation des coûts de gestion et de surveillance de l'ensemble des contrats. De ce fait, les firmes préféreront se décharger de ces activités en traitant, par exemple, avec des paysans relativement aisés qui ont accès aux circuits formels de crédit.

Face à ce risque d'exclusion des plus pauvres, l'organisation des paysans en groupements de producteurs peut constituer une réponse souhaitable du point de vue à la fois de l'efficacité et de l'équité.

* Ce travail s'appuie sur les résultats d'une étude financée par le ministère des Affaires étrangères français. Bien entendu, les commentaires et analyses développés n'engagent que leurs auteurs.

Notes :

${ }^{1}$ Entre juillet 1987 et octobre 1989, la Côte d'Ivoire a suspendu ses exportations de cacao dans le but de faire pression sur les cours mondiaux. Cette initiative de manipulation du marché mondial s'est soldée par un échec et par la mise en place d'une série de réformes.

${ }^{2}$ Maclntire et Varangis [5] ont montré que la Côte d'Ivoire a bénéficié d'une prime de terme de 1985 à 1995 environ, résultat qui va dans le sens de simulations montrant que le gain potentiel tiré des primes sur les ventes à terme n'est pas négligeable [6].

${ }^{3}$ Résultats qui rejoignent ceux du BNETD. Parmi les différents intervenants dans la commercialisation, il semblerait que les collecteurs (traitants et pisteurs) soient en position de force vis-à-vis des exportateurs et que des producteurs aient réussi à maintenir leur marge, voire à l'augmenter au cours de la première campagne libéralisée.

${ }^{4}$ À partir de la campagne 1998/1999, suite à de nombreux impayés, les banques locales exigent une plus grande surface financière de la part des exportateurs et acceptent de ne financer que les exportateurs adossés à des maisons de négoce internationales tandis que le deuxième passage, en décembre-janvier, qui se situe après le début de la campagne, est moins touché. 
${ }^{5}$ Une défaillance classique du marché des intrants découle des asymétries informationnelles entre vendeurs et acheteurs sur la qualité et la pertinence agronomique des intrants échangés. Ainsi, il est possible que des phénomènes de sélection contraire se manifestent, entraînant la disparition du marché des intrants de bonne qualité et une augmentation du prix des intrants disponibles [12].

${ }^{6}$ C'est le cas de certaines sociétés cotonnières dans des pays d'Afrique de la zone franc.

${ }^{7}$ Le PRIMAC diffuse seulement le prix moyen du jour précédent sur le marché de New York et de Londres et ne fournit aucune information sur les conditions locales du marché, autrement dit sur les écarts de prix entre le marché mondial et les principaux centres de collecte. II en résulte souvent une incompréhension des paysans qui ne voient pas le lien entre le prix affiché par le PRIMAC et le prix qui leur est offert.

${ }^{8}$ Une étude réalisée par le BNETD en 1997/1998 auprès de 336 planteurs dispersés sur l'ensemble de la zone de production montrait que $12 \%$ d'entre eux avaient contracté des crédits auprès des acheteurs de cacao en 1996/1997 et $15 \%$ auprès d'autres producteurs, parents ou amis.

${ }^{9}$ Rappelons qu'en Côte d'Ivoire, du fait de l'absence d'un véritable marché foncier, la terre n'a pas valeur de collatéral et ne facilite pas l'accès des propriétaires fonciers au crédit. Aussi, le produit (la récolte sur pied) était-il le principal collatéral à l'octroi d'un crédit.

${ }^{10}$ On estime à 30000 les producteurs concernés par la culture de l'hévéa et du palmier à huile.

${ }^{11}$ À ce prélèvement sur les exportations de fèves de cacao s'ajoutent un droit d'enregistrement fixé à $2,3 \%$ du prix CAF d'enregistrement payable au guichet unique et une redevance de $5 \mathrm{FCFA} / \mathrm{kg}$, en 2000, perçue par la Caisse.

${ }^{12}$ Le coefficient de corrélation entre les variations du DUS et celles du prix mondial exprimé en FCFA est de $82 \%$ sur la période 1990/2000.

${ }^{13}$ La réduction du DUS de 150 à 125 FCFA/kg en début de campagne 1999/2000 a été immédiatement suivie d'une baisse équivalente du prix international.

${ }^{14}$ La loi sur les coopératives, votée en décembre 1997, a pris effet en 1998, mais les GVC avaient jusqu'à la fin 2000 pour se mettre en conformité avec cette nouvelle loi.

${ }^{15}$ Les planteurs de café et cacao sont regroupés dans une demi-douzaine d'organisations syndicales ou professionnelles. La plus importante est l'APROCACI (Association des producteurs de café et de cacao) ; elle est principalement implantée au centre et à l'est du pays et regroupe 26000 planteurs et 12 coopératives, soit seulement 5 à $6 \%$ de la production nationale.

\section{CONCLUSION}

Les résultats des deux premières campagnes libéralisées tendent à montrer qu'en permettant l'entrée de nouveaux opérateurs sur le marché ivoirien, la libéralisation a été à l'origine de gains d'efficacité au niveau de la commercialisation du cacao. Néanmoins, du fait de la part relativement faible des coûts de commercialisation dans le prix d'exportation, on ne pouvait attendre de la réduction de ces coûts une amélioration importante des prix payés aux producteurs, de sorte que le revenu des planteurs s'est fortement détérioré à la suite de la chute des cours mondiaux. En raison de la position dominante de la Côte d'Ivoire sur le marché international, une réduction du prélèvement public, qui demeure 
substantiel, ne pouvait permettre d'augmenter significativement le prix au producteur, l'incidence du prélèvement étant largement reportée sur les consommateurs étrangers.

La libéralisation a eu des conséquences importantes sur le mode de coordination des agents au sein de la filière. En l'absence d'un cadre juridique garantissant le respect des contrats, la libéralisation a entraîné une forte aggravation du risque de défaut des paysans vis-à-vis des exportateurs et de ces derniers vis-à-vis des acheteurs internationaux. La perte de confiance des opérateurs a eu pour conséquence l'arrêt des transactions en différé et l'impossibilité de fixer par avance les prix aux producteurs. II en résulte une plus grande vulnérabilité des paysans dont le risque de revenu est fortement accru et dont les moyens de protection sont très limités en l'absence de mécanismes d'assurance et de crédit alternatifs. Les paysans ont été contraints de réduire certaines dépenses de consommation, notamment d'éducation, et d'exploitation. Ils ont aussi été incités à diminuer leur offre de cacao et à développer des stratégies de diversification de leurs sources de revenu. Enfin, la libéralisation s'est accompagnée d'un moindre rôle des coopératives dans la commercialisation du cacao tandis que les modalités de la participation des paysans à une autorité de régulation de la filière restent floues.

Le nouvel équilibre créé par libéralisation apparaît donc plus défavorable aux pauvres et est de ce fait probablement moins efficace. Face à l'incomplétude des réformes qui visaient essentiellement un objectif d'efficacité économique, il apparaît particulièrement important de s'attacher à mettre en place les institutions nécessaires à un meilleur fonctionnement des marchés et/ou des politiques redistributives au profit des pauvres.

\section{REFERENCES}

1. BOURGUIGNON F (2001). La redistribution peut-elle accélérer la croissance et le développement ? Rev Économie Dévelop, 1-2 : 169-96.

2. BANQUE MONDIALE (1997). Poverty in Côte d'Ivoire, a framework for action. Washington DC ; 97 p.

3. NOWAK JJ (1994). Le boom du café et du cacao en Côte d'Ivoire : une étude cas du syndrome néerlandais. Rev Économie Dévelop, 4 : 51-75.

4. ARAUJO BONJEAN C, CHAMBAS G (1999). La taxation des exportations agricoles en Côte d'Ivoire après la dévaluation. In : GASTELLU, MOISSERON, POURCET, eds. Fiscalité, Développement et Mondialisation. Paris : Maisonneuve et Larose : 159-79.

5. MACINTIRE J, VARANGIS P (1999). Reforming Côte d'Ivoire cocoa marketing and pricing system. Policy Research Working Paper ${ }^{\circ} 2081$, World Bank ; 26 p.

6. MARQUET Y (1998). Optimisation des recettes d'exportation de cacao en Côte d'Ivoire. Rev Économie Dévelop, 1 : 67-92.

7. ARAUJO C, BRUN JF, CHAMBAS C, COMBES JL, PLANE P (2001). Impact des modes d'organisation des filières agro-alimentaires dans la lutte contre la pauvreté : les filières cacao et coton. Paris : ministère des Affaires étrangères (à paraître).

8. RUF F (2000). Planteurs et chocolatiers face à face. Marchés Tropicaux, $2448: 52$. 
9. BNETD (2000). Dispositif de suivi-évaluation de la libéralisation des filières café-cacao, campagne de commercialisation 1999/2000; $33 \mathrm{p}$.

10. GOLDSMITH A (1985). The private sector and rural development: can agribusiness help the small farmer? World Develop, $13: 1125-38$.

11. KEY N, RUNSTEN D (1999). Contract farming, smallholders, and rural development in Latin America: the organization of agroprocessing firms and the scale of outgrower production. World Develop, 27 : 381-401.

12. AKERLOF G (1970). The market for lemons: qualitative uncertainty and the market mechanism. Quaterly of Economics, 84 : 488-500.

Illustrations

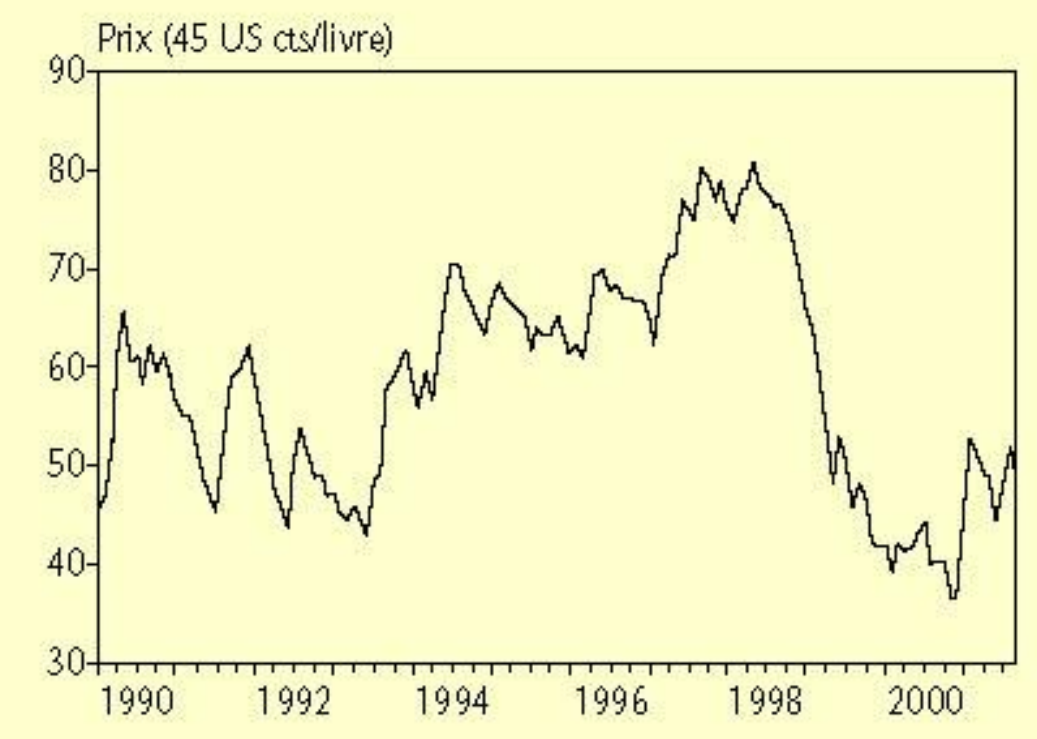

Figure. Prix mondial du cacao, indice ICCO.

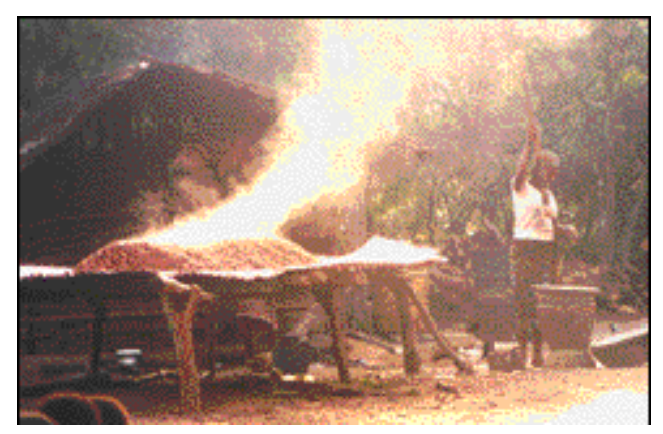

Photo. Séchage du cacao à la lisière de la forêt de Taï, Côte d'Ivoire (crédit photo : F.

Ruf). 


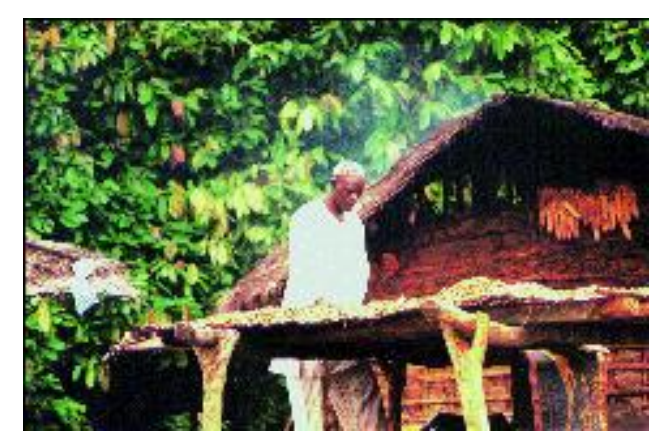

Photo. Planteur de cacao migrant, séchant son cacao (crédit photo : F. Ruf). 